\title{
Significance of Persistent Nondysplasia Over Multiple Endoscopic Surveillance in Risk Stratification of Patients With Barrett's Esophagus
} (Gastroenterology 2013;145:548-553, e1)

\author{
Jeong Hwan Kim \\ Department of Internal Medicine, Digestive Disease Center, Konkuk University School of Medicine, Konkuk University Medical Center, Seoul, \\ Korea
}

\section{Summary}

Gaddam et $\mathrm{al}^{1}$ has reported a study entitled "Persistence of nondysplastic Barrett's esophagus (BE) identifies patients at lower risk for esophageal adenocarcinoma (EAC): results from a large multicenter cohort." in the September issue of Gastroenterology 2013. Using outcomes of a large multicenter cohort study of patients with $\mathrm{BE}$, the authors evaluated how the persistence of $\mathrm{BE}$ without dysplasia over multiple consecutive surveillance using esophagogastroduodenoscopic examinations could have an effect in risk stratification of patients with BE. Based on the number of consecutive surveillance endoscopies presenting BE without dysplasia, they identified 5 groups of patients with BE without dysplasia. Group 1 was determined as the patients with BE without dysplasia at their first esophagogastroduodenoscopy (EGD). Group 2 was determined as the patients with BE without dysplasia at their first 2 consecutive EGDs. Similarly, groups 3, 4 and 5 were determined as the patients with $\mathrm{BE}$ without dysplasia at 3, 4, and 5 consecutive surveillance EGDs. A logistic regression model was used to determine whether the persistence of $\mathrm{BE}$ without dysplasia had independent effect for the development of esophageal high grade dysplasia (HGD) and/or EAC. In results, a total of 1,401 patients with BE were finally included (median follow-up period; $5.0 \pm 3.9$ years). The annual risk of HGD and/or EAC in group $1(\mathrm{n}=1,401)$, group $2(\mathrm{n}=826)$, group $3(\mathrm{n}=484)$, group $4(\mathrm{n}=280)$ and group $5(\mathrm{n}=173)$ was $0.75 \%, 0.57 \%, 0.41 \%, 0.44 \%$ and $0.00 \%$, respectively ( $P$ for trend $=0.021)$. The persistence of $\mathrm{BE}$ without dysplasia, based on multiple surveillance endoscopies, was associated with a gradually lower likelihood of progression to HGD and/or EAC (OR, $0.67 ; 95 \% \mathrm{CI}, 0.51-0.91 ; P<0.01)$, after adjusting for age, sex and length of BE. Through these results, the authors suggested that the persistence of $\mathrm{BE}$ without dysplasia over several endoscopic examinations could identify patients who are at low risk for development of HGD and/or EAC, and after all, these findings support lengthening surveillance intervals or discontinuing surveillance in the patients with persistence of $\mathrm{BE}$ without dysplasia

\section{Comment}

Barrett's esophagus is a well-established risk factor for EAC, increasing incidence of EAC at a greater rate (approximately 6-fold increase since 1975). ${ }^{2}$ The AGA guideline suggest that en-

Received: September 14, 2013 Revised: September 23, 2013 Accepted: September 24, 2013

(c) This is an Open Access article pdistributed under the terms of the Creative Commons Attribution Non-Commercial License

(http://creativecommons. org/licenses/by-nc/3.0) which permits unrestricted non-commercial use, distribution, and reproduction in any medium, provided the original work is properly cited.

*Correspondence: Jeong Hwan Kim, MD

Department of Internal Medicine, Digestive Disease Center, Konkuk University School of Medicine, Konkuk University Medical Center, 120-1, Neungdong-ro, Gwangjin-gu, Seoul 143-729, Korea

Financial support: None.

Tel: +82-2-2030-5010, Fax: +82-2-2030-5029, E-mail: sefamily@kuh.ac.kr

Conflicts of interest: None. 
doscopic surveillance would be performed in patients with BE without dysplasia as 3-5 years intervals. ${ }^{3}$ In the similar manner, the other Western guidelines recommend the enrollment of patients with BE in surveillance programs. ${ }^{4,5}$

Recent large cohort and epidemiological studies have shown that the risk of EAC in patients with $\mathrm{BE}$ without dysplasia is much lower than the assumed risk of $0.5 \%$ year, which has been the basis for current surveillance guidelines. Data from these current studies bring into question about the rationale for ongoing surveillance in patients with $\mathrm{BE}$ without dysplasia. ${ }^{6,7}$ Also, some argue lengthening the surveillance intervals or discontinuing the surveillance of patients with persistent $\mathrm{BE}$ without dysplasia. Despite the increased evidence showing a low risk of EAC in patients with $\mathrm{BE}$ without dysplasia, the inability to stratify the risk in $\mathrm{BE}$ patients has led to the ongoing recommendation for lifelong endoscopic surveillance of all patients with $\mathrm{BE}$ without dysplasia. Until now, surveillance intervals were not standardized but have been consistent with guidelines. ${ }^{7}$

In this study, Gaddam et $\mathrm{al}^{1}$ showed that the stable persistence of BE without dysplasia over several endoscopic examinations identified the patients at a very low risk of progression to EAC. The findings of this study should help to inform future decisions on surveillance intervals in patients with $\mathrm{BE}$ without dysplasia. As far as I know, this is the first study to show that patients with multiple endoscopic examinations presenting persistence of $\mathrm{BE}$ without dysplasia have a decreased risk of EAC. Despite the small sample size for groups 4 and 5 which may have resulted in a type II error and the possibility of a higher-risk cohort due to the study being performed in referral academic centers, the topic of this study is considered to be important and to have major values.

Unlike the Western countries, the Asian countries including Korea have the important distinct characteristics. First, although the prevalence of gastroesophageal reflux is increasing, the prevalence of $\mathrm{BE}$ and $\mathrm{EAC}$ has remained low in most Asian countries. $^{8-10}$ Second, upper endoscopic examination is easily accessible and available, evenly familiar in Asians, especially in Koreans, in contrast to the Western population. At present, however, there is no guideline for surveillance of $\mathrm{BE}$ in Asia, and also the data regarding the management and long-term outcome of $\mathrm{BE}$ are extremely limited in Asia. Therefore, more studies of BE in this geographic area are warranted. Furthermore, the guidelines for surveillance and management suitable for this area will be required.

\section{References}

1. Gaddam S, Singh M, Balasubramanian G, et al. Persistence of nondysplastic Barrett's esophagus identifies patients at lower risk for esophageal adenocarcinoma: results from a large multicenter cohort. Gastroenterology 2013;145:548-553, e1.

2. Pohl H, Welch HG. The role of overdiagnosis and reclassification in the marked increase of esophageal adenocarcinoma incidence. J Natl Cancer Inst 2005;97:142-146.

3. American Gastroenterological Association, Spechler SJ, Sharma P, Souza RF, Inadomi JM, Shaheen NJ. American Gastroenterological Association medical position statement on the management of Barrett's esophagus. Gastroenterology 2011;140:1084-1091.

4. Wang KK, Sampliner RE; Practice Parameters Committee of the American College of Gastroenterology. Updated guidelines 2008 for the diagnosis, surveillance and therapy of Barrett's esophagus. Am J Gastroenterol 2008;103:788-797.

5. Hirota WK, Zuckerman MJ, Adler DG, et al. ASGE guideline: the role of endoscopy in the surveillance of premalignant conditions of the upper GI tract. Gastrointest Endosc 2006;63:570-580.

6. Hvid-Jensen F, Pedersen L, Drewes AM, Sørensen HT, FunchJensen P. Incidence of adenocarcinoma among patients with Barrett's esophagus. N Engl J Med 2011;365:1375-1383.

7. Bhat $\mathrm{S}$, Coleman $\mathrm{HG}$, Yousef $\mathrm{F}$, et al. Risk of malignant progression in Barrett's esophagus patients: results from a large population-based study. J Natl Cancer Inst 2011;103:1049-1057.

8. Park JJ, Kim JW, Kim HJ, et al. The prevalence of and risk factors for Barrett's esophagus in a Korean population: A nationwide multicenter prospective study. J Clin Gastroenterol 2009;43:907-914.

9. Lee IS, Choi SC, Shim KN, et al. Prevalence of Barrett's esophagus remains low in the Korean population: nationwide cross-sectional prospective multicenter study. Dig Dis Sci 2010;55:1932-1939.

10. Chang CY, Cook MB, Lee YC, et al. Current status of Barrett's esophagus research in Asia. J Gastroenterol Hepatol 2011;26:240246 